\title{
AVALIAÇÃO DA QUALIDADE DE VIDA DE IDOSOS PRATICANTES DO MÉTODO PILATES
}

\author{
EVALUATION OF QUALITY OF LIFE IN ELDERLY WHO PRACTICE THE PILATES \\ METHOD
}

\author{
Morgana Amanda Vequi ${ }^{1}$ \\ Rosana Leal do Prado ${ }^{2}$ \\ Adriano Akira Ferreira Hino ${ }^{3}$
}

\begin{abstract}
RESUMO: Introdução: $O$ envelhecimento é o período caracterizado pelo declínio da capacidade funcional e da qualidade de vida do idoso. O método Pilates é uma técnica que pode proporcionar diversos benefícios, como fortalecimento, flexibilidade, melhora do equilíbrio e bem-estar, contribuindo assim, na qualidade de vida do idoso. Objetivo: Avaliar a qualidade de vida de idosos praticantes e não praticantes do método Pilates. Métodos: A pesquisa foi realizada com 30 idosos divididos em dois grupos de 15 sedentários (GC) e 15 praticantes de Pilates (GP). O instrumento SF-36 foi utilizado para determinar a qualidade de vida dos idosos e um questionário sociodemográfico foi aplicado. Os dados foram analisados pelo programa Epidata Analysis usando 5\% de nível de significância ( $p<0,05)$. Resultados: Os resultados demonstraram predomínio do gênero feminino, formado por aposentados com nível alto de escolaridade e renda superior a três salários mínimos em ambos os grupos. Diferenças significativas foram observadas entre os idosos sedentários e praticantes do método Pilates para os domínios: limitação funcional, aspecto emocional, estado geral de saúde, vitalidade, aspectos sociais e saúde mental após análise do questionário SF-36. Conclusão: A prática regular do Pilates é benéfica aos idosos possibilitando melhoria da qualidade de vida devido sua integração com os aspectos físicos, mentais e espirituais.
\end{abstract}

Palavras-chave: Técnicas de Exercício e de Movimento. Idosos. Qualidade de vida.

\footnotetext{
${ }^{\mathrm{I}}$ Fisioterapeuta, Especialista em Método Pilates e Ortopedia, Traumatologia e Desportiva; Mestre em Saúde Coletiva pela Fundação Universidade Regional de Blumenau/SC, Doutoranda em Saúde Coletiva pela Universidade Federal de Santa Catarina e Professora do curso de Graduação em Fisioterapia da UNIAVAN (Centro Universitário Avantis), Santa Catarina. E-mail: morganaa_vequii@hotmail.com.

${ }^{2}$ Odontóloga, Mestre em Odontologia Preventiva e Social pela Universidade Estadual Paulista Júlio de Mesquita Filho e Doutora em Odontologia Preventiva e Social pela Universidade Estadual Paulista Júlio de Mesquita Filho. Professora do departamento de Saúde Coletiva em Odontologia da Universidade do Oeste Paulista/UNOESTE e professora visitante da Fundação Universidade Regional de Blumenau (FURB).

${ }^{3}$ Graduação Licenciatura e Bacharelado em Educação Física, especialista em Fisiologia do Exercício, Mestre e Doutor em Educação Física pela Universidade Federal do Paraná. Professor no curso de graduação em Educação Física na Pontifícia Universidade Católica do Paraná na escola da Ciências da Vida e docente no Programa de Pós-Graduação em Tecnologia em Saúde e Programa de Pós-Graduação em Ciências da Saúde.
} 
ABSTRACT: Introduction: Aging is the period characterized by the decline in functional capacity and quality of life of the elderly. The Pilates method is a technique that can provide several benefits, such as strengthening, flexibility, improved balance and well-being, thus contributing to the quality of life of the elderly. Objective: Evaluate the quality of life of elderly people engaged and non-engaged in Pilates method. Methods: The research was carried out with 30 elderly people divided into two groups of 15 people each: Control group with sedentary people (GC) and Pilates group (GP). The SF-36 instrument was used to determine the quality of life of the elderly. A questionnaire to evaluate sociodemographic aspects was applied. The data were analyzed by Epidata Analysis using a 5\% level of significance ( $\mathrm{p}<0.05)$. Results: The results showed a predominance of the female gender, composed of retirees with high schooling level and income above three minimum wages in both groups. Significant differences were observed among GC and GP for the domains: functional limitation, emotional aspect, general health, vitality, social aspects and mental Morgana Amanda Vequi Fisioterapeuta, Especialista em Método Pilates e Ortopedia, Traumatologia e Desportiva; Mestre em Saúde Coletiva pela Fundação Universidade Regional de Blumenau/SC, Doutoranda em Saúde Coletiva pela Universidade Federal de Santa Catarina e Professora do curso de Graduação em Fisioterapia da UNIAVAN (Centro Universitário Avantis), Santa Catarina. E-mail: morganaa_vequii@hotmail.com.is beneficial to the elderly, enabling an improvement in the quality of life due to its integration with the physical, mental and spiritual aspects.

Keywords: Exercise Movement Techniques. Elderly. Quality of life.

\section{INTRODUÇÃO}

O envelhecimento é caracterizado por um processo universal pelo qual todos os seres humanos tendem a passar e, considerado a fase final de um todo "continuum" que é a vida. Este processo multidirecional, gradual e irreversível caracteriza-se pelo declínio das funções orgânicas com diminuição linear da capacidade funcional em função do tempo (GONÇALVES, 2015). As principais mudanças do envelhecimento são diminuição da estatura, tônus muscular, massa óssea bem como da capacidade de coordenação motora, contribuindo significativamente no aumento de quedas e possíveis fraturas (OLIVEIRA; PINTO; CORDEIRO, 2015).

O aumento da idade em idosos é o principal fator que contribui na perda muscular, aumento da obesidade e declínio funcional, muitas vezes acompanhado de fatores de risco como hipertensão, artrite/artrose e depressão que, quando tratados, podem prevenir o declínio funcional para esta faixa etária (MOREIRA et al, 20I6).

Atualmente, o envelhecimento da população é uma realidade em diversas regiões do mundo e presente até mesmo em países mais pobres. De acordo com Organização das Nações Unidas (ONU, 2013), estima-se que até 2050, mais de dois bilhões de habitantes serão idosos.

No Brasil, os números atuais reportam para aproximadamente $10,7 \%$ da população sendo considerada idosa, com projeções de aumento para até 18,7 e $29 \%$ nos anos de 2030 e 2050, respectivamente. $\mathrm{O}$ aumento significativo para o número de pessoas idosas deve-se principalmente ao aumento da população e para os índices de expetativa de vida dos brasileiros. Os números atuais indicam que entre os anos de 2020 a 2050 , a população brasileira irá aumentar de 212 para 253 milhões de habitantes enquanto, a expectativa de 
vida, irá passar de 76,7 para 8I,3 anos, respectivamente, sendo considerada a quinta maior população do planeta (LENARDT et al, 2014; IBGE, 2016).

$\mathrm{O}$ crescente número de idosos no país exige planejamento quanto aos serviços públicos especializados, assegurando cuidados para doenças crônicas da população e medidas de promoção a saúde, para garantir o envelhecimento saudável (MIRANDA; MENDES; SILVA, 20I6). Dessa forma, o envelhecimento da população é um grande desafio para a Saúde Pública, devido a dependência maior dos serviços de saúde e tempo de hospitalizações, gerando elevados custos para os serviços de Atenção à Saúde do idoso (BRITO et al, 2013).

As políticas e atividades desenvolvidas para os idosos devem levar em consideração a capacidade funcional, as necessidades de autonomia, de participação de cuidado e autossatisfação além de incentivar medidas para a prevenção, cuidado pessoal e atenção integral a saúde (MACHADO et al, 2017).

As atividades voltadas ao idoso devem exercitar o corpo e o cérebro, de maneira lúdica, descontraída e diversificada, de modo a contemplar as necessidades desta fase da vida e garantir melhor qualidade de vida aos idosos (OLIVEIRA; PINTO; CORDEIRO, 2015). Além disso, o processo de envelhecimento pode ser marcado por outras mudanças não físicas como a viuvez, problemas econômicos muitas vezes gerados por insatisfações de aposentadorias, solidão e baixa autoestima, além de alterações psíquicas do estado de saúde (MEIRELES et al, 2007), gerando preocupação constante de todos quando se busca envelhecer com qualidade de vida.

Para a Organização Mundial de Saúde (OMS) (FLECK et al, 1999) a qualidade de vida (QV) é definida como: “[...] a percepção que o indivíduo tem de sua posição na vida dentro do contexto de sua cultura e do sistema de valores de onde vive, e em relação a seus objetivos, expectativas, padrões e preocupações”. Porém, não há consenso em relação ao conceito da palavra, devido a diferenças quanto a subjetividade e complexidade nas diversas áreas de estudo em que se trata o tema, podendo muitas vezes ser considerada como sinônimo de saúde, felicidade e satisfação pessoal, condições de vida, estilo de vida, dentre outros (PEREIRA; TEIXEIRA; SANTOS, 20I2)

A busca crescente da população por qualidade de vida e bem-estar é uma lacuna complexa e ampla a ser explorada, devendo ser voltada ao equilíbrio da saúde física, social e mental de cada indivíduo. Estudos recentes demonstram que a prática de exercícios físicos, independente da faixa etária, previne doenças crônicas degenerativas e proporciona diversos benefícios a qualidade de vida dos praticantes (GONÇALVES, 2015).

$\mathrm{O}$ método Pilates é um programa completo de condicionamento físico e mental baseado na respiração e consciência corporal, formado por uma diversidade de exercícios que partem de pequenos movimentos até exercícios mais complexos e intensos (PILATES; MILLER, 1945). O método criado por Joseph Pilates é resultado de um compilado de exercícios oriundos da ginástica, yoga, artes marciais e dança (LATEY, 200I) que preconiza o fortalecimento dos músculos mais profundos da coluna vertebral para estabilização (MUSCOLINO; CIPRIANI, 2004). Além disso, o método baseia-se no equilíbrio dos exercícios e movimentos através de seis princípios básicos: respiração, controle, concentração, movimento harmônico ou fluidez de movimento, centralização e precisão (LATEY, 200I). 
$\mathrm{Na}$ literatura, diversos estudos destacam os benefícios da prática do método Pilates para a saúde do corpo, possibilitando aumento de flexibilidade e força, melhora da consciência corporal, do equilíbrio, da coordenação e capacidade funcional além de proporcionar melhor postura corporal e qualidade de vida, sendo considerada uma excelente atividade para os idosos (BERTOLDI; WINTER; FIALHO, 2016; CANCELA; OLIVEIRA; RODRIGUEZ-FUENTES, 2014; ENGERS et al, 2016). Os efeitos do método Pilates na postura, flexibilidade e força são bastante conhecidos na literatura, com inúmeros trabalhos relacionando sua prática na qualidade de vida (SINZATO et al, 2013). Todavia, informações sobre os benefícios do método Pilates na qualidade de vida de pessoas idosas ainda são limitadas, podendo ser de grande relevância na busca pelo bemestar físico, mental e social, visto as necessidades crescentes desta população em diversos países na busca por melhor qualidade de vida. O objetivo do estudo foi avaliar a qualidade de vida de idosos praticantes e não praticantes do método Pilates.

\section{METODOLOGIA}

O estudo tem caráter descritivo e utiliza uma abordagem quantitativa e observacional do tipo transversal (ARAGÃO, 20II). Foi aplicado um questionário específico para avaliação da qualidade de vida (SF-36) e um questionário sociodemográfico e de saúde. Participaram do estudo, trinta idosos de ambos os sexos, com idade equivalente ou superior a 60 anos, divididos em dois grupos com 15 idosos cada, formado por idosos praticantes de Pilates (GP) e não praticantes (GC).

Os critérios para inclusão no grupo dos praticantes do Pilates (GP) foram: idade mínima de 6o anos, não apresentar déficit de compreensão e cognição, estar lúcido e orientado e, prática do método Pilates no tempo igual ou superior há cinco meses em estúdio, na modalidade de aparelhos, realizando as aulas de maneira individual ou em duplas, duas vezes na semana, por 6o minutos cada aula. Os estúdios de Pilates foram selecionados aleatoriamente. Foi solicitado a autorização do responsável técnico de dois estúdios de Pilates já estabelecidos na cidade há mais de 4 anos. Enquanto que, para o grupo dos não praticantes (GC), foram utilizados os mesmos critérios do GP, exceto para prática do método Pilates, tendo sido incluídos neste grupo idosos que não realizavam nenhuma atividade física regularmente. Estes idosos por sua vez, foram abordados de forma aleatória em um município do sul do país, após indicação dos praticantes de Pilates sobre pessoas idosas sedentárias que estes conheciam. Foram realizados contatos telefônicos e mediante agendamento, realizou-se a coleta de dados em suas residências, de maneira individual. Todos os idosos que não apresentaram os critérios mínimos exigidos foram excluídos deste estudo.

A coleta de dados foi realizada no período de janeiro a junho de 2015, com aplicação do questionário sociodemográfico e de saúde, contendo as seguintes questões: nome, sexo, endereço, telefone, data de nascimento, idade, naturalidade, profissão/ocupação, situação profissional, escolaridade, renda familiar, doenças referidas, hábitos sociais e de vida, com quem mora. O tempo previsto para a realização do questionário sociodemográfico foi de aproximadamente dez minutos e; e o instrumento SF-36 (Short Form-36), validado em 
português para avaliar a qualidade de vida dos idosos foi aplicado em tempo estimado de is minutos.

$\mathrm{O}$ instrumento SF-36 é composto por il questões e 36 itens que englobam oito componentes sobre domínios ou dimensões, representados por capacidade funcional (dez itens), aspectos físicos (quatro itens), dor (dois itens), estado geral da saúde (cinco itens), vitalidade (quatro itens), aspectos sociais (dois itens), aspectos emocionais (três itens), saúde mental (cinco itens) e uma questão comparativa sobre a percepção atual da saúde e há um ano (WARE; KOSINSKI; GANDEK, 2003)

O entrevistado recebeu um escore para cada domínio, com notas variando de "zero a cem", onde zero refere-se ao menor escore enquanto que o valor cem foi atribuído para o melhor (IBGE, 2018). Ambos os questionários foram aplicados pela própria pesquisadora em forma de entrevista. Os indivíduos pertencentes aos GC e GP e, que concordaram espontaneamente em participar do estudo, assinaram o Termo de Consentimento Livre e Esclarecido, conforme resolução $466 / 12$ do Comitê de Ética e Pesquisa, sob parecer número: 897.092. Os dados desta pesquisa foram resguardados quanto a privacidade $\mathrm{e}$ confiabilidade, preservando integralmente o anonimato dos sujeitos.

\section{ANÁLISE DOS DADOS}

Os dados da análise descritiva obtido pelos questionários sociodemográficos e de saúde a fim de avaliar a qualidade de vida dos idosos, foram ajustados conforme as orientações contidas no The SF-36 $6^{\circledast}$ - Health Survey Manual. Inicialmente, foi estabelecida a correspondência para cada item ou questão à sua respectiva escala.

Os dados de qualidade de vida foram então computados, considerando os valores recodificados e, os escores dos itens de cada escala, foram somados para corresponder às respostas dos entrevistados. Após isso, o valor de cada escore foi transformado para a escala de "zero a Ioo", seguindo as orientações para somatória dos pontos e, foi aplicado o cálculo do Raw Scale. Esta transformação compreendeu a conversão do valor mínimo e máximo possível de cada item padronizado para cálculo das escalas transformadas em cada escala e para cada paciente. Os escores entre estes valores representaram a porcentagem possível de ser alcançada de qualidade de vida para cada um dos oito domínios envolvidos, sendo diretamente proporcional a porcentagem.

Após isso, os dados obtidos foram analisados pelo programa Epidata Analysis versão 2.2.2.183 e, as médias dos dados em cada grupo avaliado, foi comparado pelo teste $t$ de Student utilizando um nível de significância de $5 \%$ ( $\mathrm{p}<0,05)$.

\section{RESULTADOS}

Os resultados descritivos deste estudo para o questionário sociodemográfico e de saúde em idosos praticantes do método Pilates (GP) e sedentários (GC) estão descritos na Tabela I. Ao avaliar a idade dos idosos, houve homogeneidade estatística entre os grupos avaliados, em que a idade média foi de $67,47( \pm 6,95)$ anos para o GP e de $68,87( \pm 5,04)$ anos para GC (dados não apresentados), com predomínio do gênero feminino em ambos os grupos.

Tabela I - Estatística descritiva dos idosos sedentários e praticantes do método Pilates ( $\mathrm{n}=30)$ 


\begin{tabular}{|c|c|c|c|c|}
\hline \multirow{2}{*}{ Item do questionário } & \multicolumn{2}{|c|}{ Idosos sedentários } & \multicolumn{2}{|c|}{ Idosos praticantes de Pilates } \\
\hline & $\mathbf{N}$ & $\%$ & $\mathbf{N}$ & $\%$ \\
\hline \multicolumn{5}{|l|}{ Gênero } \\
\hline Feminino & Io & 66,6 & Io & 66,6 \\
\hline Masculino & 5 & 33,3 & 5 & 33,3 \\
\hline Trabalha & 6 & 40 & 3 & 20 \\
\hline \multicolumn{5}{|l|}{ Situação profissional } \\
\hline Aposentado & II & 73,3 & 14 & 93,3 \\
\hline Trabalha com registro & 3 & 20 & I & 6,7 \\
\hline Trabalho informal & I & 6,7 & o & o \\
\hline \multicolumn{5}{|l|}{ Escolaridade } \\
\hline Fundamental incompleto & 4 & 26,7 & o & o \\
\hline Fundamental completo & I & 6,7 & o & o \\
\hline Ensino médio incompleto & 3 & 20 & o & o \\
\hline Ensino médio completo & 2 & 13,3 & 4 & 26,7 \\
\hline Superior incompleto & I & 6,7 & 3 & 20 \\
\hline Superior completo & 4 & 26,7 & 7 & 46,7 \\
\hline Outro & o & o & I & 6,7 \\
\hline \multicolumn{5}{|l|}{ Renda } \\
\hline Até um salário-mínimo & 2 & 13,3 & I & 6,7 \\
\hline De I até 3 salários-mínimos & 6 & 40 & o & o \\
\hline De 3 até 5 salários-mínimos & 3 & 20 & 5 & 33,3 \\
\hline Acima de 5 salários-mínimos & 4 & 26,7 & 9 & 60 \\
\hline \multicolumn{5}{|l|}{ Patologias referidas } \\
\hline Hipertensos & 8 & 53,3 & 4 & 26,7 \\
\hline Diabetes Mellitus & o & o & 2 & 13,3 \\
\hline Osteoporose & I & 6,7 & 2 & 13,3 \\
\hline Doença de Parkinson & I & 6,7 & o & 0 \\
\hline Síndromes cerebelares & I & 6,7 & o & o \\
\hline Alterações visuais & o & o & 3 & 20 \\
\hline Distúrbios osteomioarticulares & 3 & 20 & o & o \\
\hline Outras & 3 & 20 & 2 & 13,3 \\
\hline \multicolumn{5}{|l|}{ Prática de Pilates ( $x$ por semana) } \\
\hline Nenhum & 15 & IOO & o & o \\
\hline Um dia & o & o & 3 & 20 \\
\hline Dois & o & o & 7 & 46,7 \\
\hline Três & o & o & 5 & 33,3 \\
\hline \multicolumn{5}{|l|}{ Tabagismo } \\
\hline Ex-tabagista e/ou não fumante & 2 & 13,3 & I & 6,7 \\
\hline Fumante & I & 6,7 & 2 & 13,3 \\
\hline Etilista & I & 6,7 & o & o \\
\hline \multicolumn{5}{|l|}{ Reside } \\
\hline Sozinho & 3 & 20 & 3 & 20 \\
\hline Marido/mulher/companheiro (a) & 6 & 40 & Io & 66,7 \\
\hline Companheiro e animais de estimação & 6 & 40 & I & 6,7 \\
\hline Outros parentes & o & $\mathrm{o}$ & I & 6,7 \\
\hline
\end{tabular}

Para os demais itens do questionário, os dados demonstraram que o GP é caracterizado, em sua maioria, por indivíduos aposentados que apresentam alto nível de escolaridade e renda superior a três salários-mínimos, com prática de Pilates pelo menos duas vezes por semana. Para o GC, os dados coletados indicam que, pelos menos $40 \%$ dos idosos ainda exercem algum tipo de trabalho e, mais de $50 \%$ possuem escolaridade inferior 
ao ensino médio e salário menor que cinco mínimos. Ao avaliar as questões sobre patologia, $53 \%$ dos idosos entrevistados do GC são hipertensos enquanto que, para o GP, este valor é $26,7 \%$ (Tabela I). Em ambos os grupos, apenas $20 \%$ dos idosos residem sozinhos além de evidenciar resultados positivos quanto ao uso de tabaco, com valores inferiores a $14 \%$.

Em relação à qualidade de vida avaliados pelo questionário $\mathrm{SF}-36$, a pontuação obtida para cada um dos grupos nas oito dimensões em GC e GP estão descritos na Tabela 2. A análise dos dados evidenciou diferença estatisticamente significativa nas dimensões capacidade funcional $(p=0,049)$, limitação por aspectos físicos $(p=0,0001)$, estado geral de saúde $(\mathrm{p}=\mathrm{o}, \mathrm{o2} \mathrm{o})$, aspectos sociais $(\mathrm{p}=\mathrm{o}, \mathrm{oor})$, limitação por aspectos emocionais $(\mathrm{p}=\mathrm{o}, 008)$ e saúde mental $(p=0,00 I)$ enquanto que, para o domínio Vitalidade $(p=0,112)$, não houve diferença mínima significativa (Tabela 2 ).

Tabela 2 - Relação dos valores médios dos domínios avaliados pelo instrumento SF-36 nos praticantes de Pilates e sedentários não praticantes de Pilates $(n=30)$

\begin{tabular}{lcc}
\hline \multicolumn{1}{c}{ Domínios } & $\begin{array}{c}\text { Idosos praticantes de } \\
\text { Pilates }\end{array}$ & Idosos sedentários \\
\hline Capacidade Funcional & $81,3^{*}$ & 67,6 \\
Limitação por aspectos físicos & $100{ }^{*}$ & 48,3 \\
Dor & $72^{*}$ & 59,6 \\
Aspecto geral de saúde & $67,3^{*}$ & 54,7 \\
Vitalidade & $70,7^{\mathrm{ns}}$ & 63 \\
Aspectos sociais & $92,3^{*}$ & 74 \\
Limitação por aspectos emocionais & $\mathrm{IOO}^{*}$ & $53, \mathrm{I}$ \\
Saúde mental & $82, \mathrm{I}$ & $62, \mathrm{I}$ \\
\hline Média da qualidade de vida & 83,2 & 60,3 \\
\hline *Significativo estatisticamente pelo teste $\mathrm{t}$ de Student $(\mathrm{p}<0,05) .{ }^{\mathrm{n}}{ }^{*}$ não significativo.
\end{tabular}

Os melhores valores do questionário $\mathrm{SF}-36$ foram observados no GP para os todos domínios, com destaque para limitação por aspectos físicos e limitação por aspectos emocionais com média de Ioo,oo $( \pm 0,0)$, aspectos sociais com média de 92,33 ( $\pm 11,54)$, saúde mental com 82,13 ( $\pm 11,80)$ e capacidade funcional onde a média foi de $81,33( \pm 18,56)$, exceto para vitalidade, onde os valores médios entre o GP e GC foi estatisticamente similares (Tabela 2). Ao avaliar o escore final da qualidade de vida, notou-se que os idosos praticantes de Pilates atingiram a média de 83,20, sendo classificado como "Ótima qualidade de vida". Já, para os idosos sedentários, o escore final de qualidade de vida foi de 6o,30, considerado na classificação como "Boa qualidade de vida".

\section{DISCUSSÃO}

Ao avaliar a qualidade de vida de idosos praticantes e não praticantes do Método Pilates, os resultados demonstraram que mulheres são maioria na prática da atividade em comparação com os homens (ENGERS et al, 2016). A maior expectativa de vida entre 
idosas é algo comum tanto em nível mundial como no Brasil, em que as mulheres idosas representam 16,9 milhões, cerca de 56\%, enquanto há 13,3 milhões de homens na mesma faixa etária, evidenciando a feminização na velhice (IBGE, 2018). Destaca-se a necessidade de um olhar mais atento para elaboração de medidas de promoção a saúde de idosos, ressaltada pelo envelhecimento populacional decorrente da transição demográfica (ALMEIDA et al, 2015). Foi observado elevado nível de escolaridade em ambos os grupos. Porém, entre os praticantes de Pilates a escolaridade foi mais elevada, o que evidencia a busca de melhor qualidade de vida por pessoas com maior instrução, dada a procura pela prática de atividade física (REIS; MASCARENHAS; LYRA, 20II).

$O$ percentual de idosos que ainda exercem algum tipo de trabalho para complementar renda foi elevado, englobando aproximadamente 30\% do número total de entrevistados. A complementação da renda por alguns idosos mesmo após aposentados, visa manter o padrão de vida habitual e a ocupação com atividades que possibilitam o bemestar (CARTAXO et al, 2012). Todavia, para a variável renda, os resultados evidenciaram que 6o\% do GP possui renda superior a cinco salários mínimos enquanto que, a renda para mais de $73 \%$ do GC, é inferior a cinco salários mínimos. Há uma correlação entre renda e qualidade de vida, em que idosos com menor poder aquisitivo tendem a apresentar piores condições de saúde e, consequentemente, menor qualidade de vida (GORGIA et al, 2007; SOUZA; GALANTE; FIGUEIREDO, 2003). Além disso, acredita-se que a diferença de renda para idosos praticantes de Pilates, ocorra devido a prática personalizada e, muitas vezes, individualizada, tornando o valor das sessões mais elevado quando comparada a outras modalidades esportivas. Dessa forma, a renda possui relação direta com a prática do método Pilates, podendo limitar o acesso e benefícios que o método oferece.

Em relação as comorbidades, a maioria dos idosos pertencentes a ambos os grupos apresentou hipertensão arterial sistêmica ou algum outro tipo de patologia. A ocorrência de doenças crônicas tende a aumentar com o passar do tempo, acarretando incapacidades e limitações funcionais nos idosos (REIS; MASCARENHAS; LYRA, 2oIr; LIPOSCKI et al, 2016). Doenças crônicas não transmissíveis constituem um problema de saúde entre os idosos devido a maus hábitos de vida e a falta de adesão a tratamentos adequados, podendo gerar lesões ao organismo e aumentar as chances de incapacidades, especialmente para hipertensão arterial considerado um fator de risco importante para doenças cardiovasculares em idosos (FARIAS; SANTOS, 2012).

A prática do método Pilates contribui age sobre vários aspectos patológicos, na manutenção e melhora no tempo de realização das atividades de vida do cotidiano, além de ser considerada uma técnica segura, desde que bem orientada por um profissional habilitado, pois a possibilidade de lesões ou dores musculares é praticamente inexistente, visto que o impacto nas articulações durante o exercício é nulo (CURI; HAAS; WOLFF, 2014).

Em ambos os grupos deste estudo, o uso de tabaco foi inferior a $14 \%$, sendo possível observar maior número de fumantes entre homens idosos com nível de escolaridade mais baixo, aliado ao maior uso de álcool (SENGER et al, 20II). O abuso de substâncias como tabaco e álcool tornam os idosos mais vulneráveis devido às diversas consequências que exercem no meio familiar e social, e reforça a necessidade de adoção de medidas de prevenção (SENGER et al, 20II). 
Devido a menor longevidade do homem, muitas mulheres se tornam viúvas e/ou sozinhas (ROSA et al, 2013). Muitas idosas sentem a necessidade de uma nova companhia, seja ela pessoa ou até mesmo animais de estimação. Os dados de um estudo conduzido em Belo Horizonte-MG demonstraram que $98 \%$ dos idosos moravam na companhia de alguém (BORGES et al, 2008). A maior longevidade das mulheres, por vezes pode ser acompanhada por problemas de saúde, advindos de hábitos de vida inadequados (MATTOS et al, 2015). Logo, a qualidade de vida e bem-estar do idoso deve ser visto com cuidado, podendo ser um reflexo dos hábitos do cotidiano, mas que podem ser minimizadas pela adoção de políticas públicas e de saúde coletiva voltadas para os habitantes desta faixa etária (GONÇALVES, 2015).

Os melhores valores para qualidade de vida em todos os domínios observados no GP através do questionário SF-36, demonstram que o método Pilates pode ser considerado uma atividade importante para o bem-estar do idoso. Praticantes do Pilates apresentaram escores maiores em relação ao grupo sedentário com diferenças estatísticas significativas nas dimensões de "estado geral de saúde" e "vitalidade" (BORGES et al, 2008; NASCIMENTO; DE CARVALHO, 20r6). Adicionalmente, o método Pilates exerce influências positivas na qualidade de vida possibilitando diminuição do quadro álgico, maior flexibilidade e menor dor em idosos praticantes comparado a idosos sedentários (TOZIM et al, 2014).

Dessa forma, a prática do método Pilates pode contribuir na melhora da qualidade de vida dos idosos, proporcionando diversos benefícios físicos, mentais e sociais (REIS; MASCARENHAS; LYRA, 20II). Além de uma diminuição significativa do tempo para a realização das atividades de vida diária dos idosos (CURI; HAAS; WOLFF, 2014). De maneira similar, a prática regular do Método Pilates duas vezes na semana trouxe benefícios ao bem-estar e a qualidade de vida dos idosos, tornando uma prática cada vez mais presente no dia-a-dia do idoso (SANCHEZ; BRASIL; FERREIRA, 2014).

A influência positiva do Método Pilates na aptidão física dos idosos, bem como seus efeitos na aptidão neuromotora, equilíbrio estático e dinâmico, o tornam recomendado como modalidade de exercício ideal para melhorar o equilíbrio em idosos (CANCELA; OLIVEIRA; RODRIGUEZ-FUENTES, 2014). Quando avaliado a influência do Método Pilates sobre a capacidade funcional, qualidade de vida e remodelação óssea de idosas, foi observado melhor escore de qualidade de vida e melhora significativa da capacidade funcional. Além disso, os aspectos físicos, emocionais e a vitalidade são maiores em praticantes regulares de Pilates, com ganhos significativos (GANDOLFI et al, 2019).

O método Pilates mostra-se eficaz na promoção da percepção da qualidade de vida de mulheres sexagenárias e septuagenárias, por melhorar os escores dos oito domínios do instrumento SF-36 (NASCIMENTO et al, 2018).

Com o passar dos anos ocorre limitações no desempenho motor, alterando significativamente a capacidade de realizar as atividades de vida diárias e consequentemente a qualidade de vida dos idosos, a prática de atividade física regular, tais como o Pilates proporciona melhora da estabilidade corporal e desempenho motor promovendo maior autonomia ao idoso (RODRIGUES et al, 20I0). Além disso, é de suma 
importância que o idoso se mantenha ativo, pois além de diversos benefícios a atividade física previne doenças crônicas degenerativas, comuns nesta população (WHO, 2010).

O método Pilates em aparelhos é um tratamento personalizado, que contribui para melhorar a qualidade de vida de idosos, pois promove bem-estar físico, psicológico, social e espiritual, diminui a dor, o desconforto, a fadiga e recupera a mobilidade, proporcionando melhora na realização das atividades da vida diária (COSTA et al, 2or8). Além disso, contribui para a saúde psicológica, sentimentos positivos, concentração, memória, autoestima, imagem corporal, espiritualidade, as crenças pessoais e as relações sociais (COSTA et al, 2or8).

A prática do Pilates traz melhora significativa na qualidade de vida de idosos praticantes do método, por atuar no fortalecimento do centro de força, estabilização da coluna, melhorar a postura e diminuir o gasto energético, o que gera benefícios e melhora o equilíbrio dos praticantes, prevenindo as quedas, atingindo também uma melhora psíquica e sócio efetiva (FREITAS; SOUZA, 2019).

A independência na realização das atividades de vida diária fica comprometida no idoso, provocando impactos negativos na qualidade de vida, uma vez que que praticantes regulares de Pilates preservam por mais tempo e com maior qualidade sua independência e autoestima, por força muscular de maneira global, o que reduz os efeitos do envelhecimento (DELLA TORRE et al, 2017).

A renda, o baixo uso de medicações e a diminuição de limitações contribui para a percepção positiva de idosos com relação a qualidade de vida (JR SANTOS et al, 2016), a presença de comorbidade e renda baixa interfere na funcionalidade, tornando o idoso mais dependente (ALMEIDA et al, 2017). No estudo os praticantes de Pilates possuíam maior renda, maior escolaridade e menor número de comorbidades.

De acordo com esta pesquisa, verifica-se que o Pilates foi capaz de oferecer benefícios à essa população, pois as médias obtidas entre os voluntários praticantes do método Pilates, foram maiores em todos os domínios avaliados pelo questionário do instrumento SF-36. Dessa forma, a realização de atividade física e em especial o método Pilates, têm papel importante na melhora da qualidade de vida do idoso. Uma das limitações do presente estudo está na sua natureza transversal, que nos impossibilita o estabelecimento de relação causal, por exemplo sobre o efeito da atividade física nas comorbidades.

\section{CONCLUSÃO}

Com o presente estudo verificou-se que a prática regular do Pilates com aparelhos trouxe ganho na qualidade de vida aos idosos praticantes quando comparados com idosos sedentários. Isso pode ser devido a integração dos aspectos físicos, mentais e espirituais com melhora significativa da qualidade de vida, principalmente nos domínios limitação funcional, aspecto emocional, estado geral de saúde, aspectos sociais e saúde mental. Foi possível verificar também que os praticantes de Pilates possuíam maior renda, escolaridade e menor prevalência de comorbidades, fatores que favorecem melhor qualidade de vida e a prática de atividade física. $O$ domínio vitalidade não apresentou significância, mas 
apresentou escore maior dentre os praticantes da atividade comparado ao grupo de idosos sedentários.

A carência de informações nesta área da saúde, evidencia a necessidade de novos estudos sobre a qualidade de vida em idosos praticantes de Pilates, de modo a elucidar as possíveis contribuições da prática sobre o bem-estar de vida do idoso.

\section{REFERÊNCIAS}

ALMEIDA AV, et al. A feminização da velhice: em foco as características socioeconômicas, pessoais e familiares das idosas e o risco social. Revista Textos d Contextos - Porto Alegre; I4(I):115-131, 2015.

ALMEIDA P, et al. Funcionalidade e fatores associados em idosos participantes de grupo de convivência. Revista da Associação Brasileira de Atividade Motora Adaptada, Marília; I8(I): 53-64, 2017 .

ARAGÃO J. Introdução aos estudos quantitativos utilizados em pesquisas científicas. Revista Práxis; 3 (6): 59-62, 2011.

BERTOLDI JT; WINTER RA; FIALHO SP. Efeitos do Método Pilates na mobilidade da coluna vertebral e na qualidade de vida de idosos: estudo de caso. Revista Cinergis; $17(\mathrm{I}): 22-26,2016$.

BORGES PLC, et al. Perfil dos Idosos Frequentadores de Grupos de Convivência em Belo Horizonte, Minas Gerais, Brasil. Cadernos de Saúde Pública; 24(I4):2798-28o8, 2008.

BRITO MCC, et al. Envelhecimento populacional e os desafios para a saúde pública: análise da produção científica. Revista Kairos; 16(2):161-78, 2013.

CANCELA JM; OLIVEIRA IM; RODRIGUEZ-FUENTES G. Effects of Pilates method in physical fitness on older adults: a systematic review. European Review of Aging and Physical Activity; II(2):8I-94, 2014.

CARTAXO HGO, et al. Percepção de idosas sobre o envelhecimento com qualidade de vida: subsídio para intervenções públicas. Revista Rene; I3(I):158-68, 2012. 
COSTA TRA, et al. Comparação da percepção da qualidade de vida em idosas praticantes e não praticantes do Método Pilates. Cadernos de Saúde Coletiva; Rio de Janeiro; 26(3): 26I-269, 2018.

CURI PVS; HAAS NA; WOLFF SS. Analysis of activities in the daily lives of older adults exposed to the pilates method. Journal of Bodywork and Movement Therapies; 18:326 31.26, 2014 .

DELLA TORRE AP, et al. A influência do método Pilates no público idoso: uma revisão de literatura. Revista Pesquisa e Ação; 3(2):58-72, 2017.

ENGERS PB, et al. Efeitos da prática do método Pilates em idosos: uma revisão sistemática. Revista Brasileira de Reumatologia; 56(4):352-365, 2016.

FARIAS RG; SANTOS SMA. Influência dos determinantes do envelhecimento ativo entre idosos mais idosos. Revista Texto Contexto Enfermagem; 21(I):167-176, 2012.

FLECK MPA, et al. Desenvolvimento da versão em português do instrumento de avaliação de qualidade de vida da OMS (WHOQOL-ıoo). Revista Brasileira de Psiquiatria, v. 2I, n. I, p. 19-28. 1999.

FREITAS JP; SOUZA AT. Avaliação da qualidade de vida de idosos praticantes e não praticantes do método Pilates em aparelhos. Revista Inspirar movimento e saúde; i6 (3):19p, 2019

GANDOLFI NRS, et al. The influence of the Pilates method on quality of life and bone remodelling in older women: a controlled study. Quality of Life Research, 2019. https://doi.org/10.1007/sini36-019-02293-8.

GONÇALVES CD. Envelhecimento bem-sucedido, envelhecimento produtivo e envelhecimento ativo: reflexões. Estudos Interdisciplinares sobre o Envelhecimento, 20(2):645657,2015 .

GORGIA AP, et al. Comparação da qualidade de vida de mulheres idosas praticantes e não praticantes de exercício físico. Revista Digital-Buenos Aires; II(Io6):I-I2, 2007.

INSTITUTO BRASILEIRO DE GEOGRAFIA E ESTATÍSTICA (IBGE). Longevidade Humana e Taxa de Fecundidade, Natalidade. 2016. Disponível em: 
http://www.ibge.gov.br/home/estatistica/populacao/tabuadevida/evolucao_da_mortali dade.shtm. Acesso em: ro out. 2018.

INSTITUTO BRASILEIRO DE GEOGRAFIA E ESTATÍSTICA (IBGE). Número de idosos cresce $18 \%$ em 5 anos e ultrapassa 30 milhões em 2017. Rio de Janeiro: IBGE; 2018. Disponível em: https://agenciadenoticias.ibge.gov.br/agencia-noticias/2012agencia-de-noticias/noticias/20980-numero-de-idosos-cresce-18-em-5-anos-e-ultrapassa30-milhoes-em-2017. Acesso em: or nov. 2018.

JR SANTOS, et al. Qualidade de Vida em Idosos de Baixa Renda. Revista Motricidade; I2(2):139-I46, 2016.

LATEY, P. The Pilates Method: History and Philosophy. Journal of Bodywork Movement Therapies. 5(4): 275-82, 2001.

LENARDT MH, et al. Qualidade de vida de idoso fragilizado da atenção primária. Acta Paulista de Enfermagem, 27(5):399-404, 2014.

LIPOSCKI DB, et al. Aptidão motora de idosos longevos - implicações cognitivas e socioemocionais. Revista Kairós Gerontologia; I9 (22): 227-239, 2016.

MACHADO DW, et al. Elderly with not transmitted chronic diseases: a group association study. Revista Ciência dr Saberes; 3(2):444-45I, 2017.

MATTOS AIS, et al. Desigualdades de gênero: uma revisão narrativa. Revista Saúde.Com; iI(3):266-279, 2015.

MEIRELES VC, et al. Característica dos idosos em área de abrangência do Programa Saúde da Família na região noroeste do Paraná: contribuição para a gestão do cuidado em enfermagem. Revista Saúde e Sociedade; I6(I):69-80, 2007.

MIRANDA GMD; MENDES ACG; SILVA ALA. O envelhecimento populacional brasileiro: desafios e consequências sociais atuais e futuras. Revista Brasileira de Geriatria e Gerontologia; 19(3):507-19, 2016. 
MOREIRA PL, et al. Anthropometric, functional capacity, and oxidative stress changes in Brazilian community-living elderly subjects. A longitudinal study. Archives of Gerontology and Geriatrics, 66(6):140-146, 2016.

MUSCOLINO J; CIPRIANI, S. Pilates and "Powerhouse" I. Journal of Bodywork Movement Therapies. 8:15-24, 2004.

NASCIMENTO MM, et al. Efeitos da prática regular do método Pilates sobre a percepção da qualidade de vida de mulheres sexagenárias e septuagenárias. Revista Brasileira de Qualidade de Vida; 10(2):1-16, 2018.

NASCIMENTO MM; DE CARVALHO ECF. A percepção da qualidade de vida de septuagenárias praticantes do método pilates. Revista CPAQV-Centro de Pesquisas Avançadas em Qualidade de Vida; 8(3):1-9, 2016.

OLIVEIRA DA; PINTO NS; CORDEIRO LS. Psicomotricidade na senescência. Revista Interdisciplinar do Pensamento Científico, I(20):106-194, 2015.

ONU- Department of Economic and Social Affairs. Population Division.World Population Ageing. 2013. Disponível em: http://www.un.org/en/development/desa/population/publications/pdf/ageing/World PopulationAgeing2013.pdf Acesso 22 out. 2018.

PEREIRA EF; TEIXEIRA CS; SANTOS A. Qualidade de vida: abordagens, conceitos e avaliação. Revista Brasileira de Educação Física e Esporte; 26(2):241-250, 2012.

PILATES JH; MILlER WJ. Return to Life through Contrology. Presentation Dynamics, Incline Village, Nevada. 1945.

REIS LA; MASCARENHAS CHM; LYRA JE. Avaliação da qualidade de vida em idosos praticantes e não praticantes do método pilates. Revista Eletrônica da Fainor; 4(I):385I, 20II.

RODRIGUES BGS, et al. Autonomia funcional de idosas praticantes de Pilates. Revista Fisioterapia e Pesquisa;17(4): 300-5, 2010. 
ROSA KB, et al. Qualidade de vida e avaliação funcional em idosos praticantes de pilates e idosos sedentários. Revista Interdisciplicar de Estudos em Saúde; 2(I):18-28, 2013.

SANCHEZ MA; BRASIL JMM; FERREIRA IAM. Benefícios de um programa de atividade física para a melhoria da qualidade de vida de idosos no estado do Rio de Janeiro. Revista Brasileira de Ciências do Envelhecimento Humano; II(3):209-218, 2014.

SENGER AEV, ET AL. Alcoolismo e tabagismo em idosos: relação com ingestão alimentar e aspectos socioeconômicos. Revista Brasileira de Geriatria e Gerontologia; I4(4):713-719, 2011.

SINZATO C, et al. Efeitos de 20 sessões do método Pilates no alinhamento postural e flexibilidade de mulheres jovens: estudo piloto. Revista Fisioterapia E Pesquisa; 20(2):I43150, 2013 .

SOUZA L; GALANTE H; FIGUEIREDO D. Qualidade de Vida e bem-estar dos idosos: um estudo exploratório na população portuguesa. Revista Saúde Pública; 37(3):364371, 2003.

TOZIM BM, et al. Efeito do método Pilates na flexibilidade, qualidade de vida e nível de dor em idosos. Revista ConScientiae Saúde;13(4):563-570, 2014.

WARE JE; KOSINSKI M; GANDEK B. SF-36 Health Survey: Manual \& Interpretation Guide. Lincoln, RI: QualityMetric Incorporated, 2003.

WORLD HEALTH ORGANIZATION (WHO). Global recommendations on physical activity for health. Geneva: WHO; 2010. 